\title{
Multilinguales
}

\section{Insécurité linguistique en contexte minoritaire algérien : enquête sociolinguistique auprès des locuteurs de Tasahlit}

Linguistic insecurity in an Algerian minority context : sociolinguistic survey among the Tasahlit speakers

\section{Bachir Bessai}

\section{OpenEdition}

Journals

Édition électronique

URL : https://journals.openedition.org/multilinguales/4102

DOI : $10.4000 /$ multilinguales.4102

ISSN : 2335-1853

\section{Éditeur}

Université Abderrahmane Mira - Bejaia

\section{Référence électronique}

Bachir Bessai, «Insécurité linguistique en contexte minoritaire algérien : enquête sociolinguistique auprès des locuteurs de Tasahlit », Multilinguales [En ligne], 11 | 2019, mis en ligne le 17 juillet 2019, consulté le 21 septembre 2021. URL : http://journals.openedition.org/multilinguales/4102 ; DOI :

https://doi.org/10.4000/multilinguales.4102

Ce document a été généré automatiquement le 21 septembre 2021.

\section{(c) $($ () $\ominus$}

Multilinguales est mise à disposition selon les termes de la Licence Creative Commons Attribution -

Pas d'Utilisation Commerciale - Pas de Modification 4.0 International 


\title{
Insécurité linguistique en contexte minoritaire algérien : enquête sociolinguistique auprès des locuteurs de Tasahlit
}

\author{
Linguistic insecurity in an Algerian minority context : sociolinguistic survey \\ among the Tasahlit speakers
}

Bachir Bessai

1 Notre recherche se propose de réfléchir à la notion d'insécurité linguistique et de tenter, grâce à l'étude des représentations linguistiques, d'examiner les manifestations de ce phénomène chez des locuteurs de la région du Sahel. L'approche que nous privilégions dans le cadre de ce travail est l'étude des représentations des locuteurs en faisant fi de leurs pratiques langagières effectives. Nos enquêtés étaient amenés à répondre à un certain nombre de questions en produisant un discours épilinguistique. L'analyse de ces discours nous conduira à dégager les nombreuses attitudes, positives ou négatives, qu'adoptent les locuteurs à l'égard de leur variété -ou sociolecte- et de révéler ainsi les multiples facettes de l'insécurité linguistique, notamment la dépréciation de leurs usages linguistiques. Notons que l'insécurité linguistique est une caractéristique de bien des locuteurs des langues (ou variétés de langue) minoritaires. Nous verrons d'ailleurs que le tasahlit, que l'on peut considérer comme une variété minorée et minoritaire, fait face à une certaine dévalorisation par ses propres locuteurs.

2 Nous commencerons d'abord par une brève présentation du cadre théorique de notre recherche, nous exposerons la situation linguistique de notre population d'enquête, puis nous expliciterons le cadre méthodologique qui a été privilégié, et enfin, nous aborderons de manière plus détaillée, la question des représentations linguistiques chez des locuteurs du Sahel en insistant sur les divers signes de l'insécurité linguistique. En effet, nous mettrons l'accent sur l'insécurité linguistique « dite » et non sur l'insécurité linguistique "agie» pour reprendre la terminologie de Marie-Louise 
Moreau. La première est perçue au travers des discours épilinguistiques, chargés de stéréotypes, sous-tendus par l'idéologie dominante. La seconde est perçue au travers des pratiques, des comportements langagiers comme le bégaiement, la répétition, les coupures, la reformulation, l'hésitation, le silence, l'hypercorrection, l'alternance codique, les stratégies d'évitement ou au contraire d'exposition, etc. (Hafez, 2012)

\section{Quelques repères théoriques}

3 Notre recherche s'inscrit dans le cadre général de la sociolinguistique en explorant deux concepts clés : la représentation linguistique et l'insécurité linguistique. On parle généralement des représentations linguistiques pour désigner les diverses images mentales que se font les locuteurs des langues, des pratiques langagières, de leurs légitimités, etc.

De nos jours, la notion de représentation linguistique occupe une place importante dans toute approche sociolinguistique. Elle est de plus en plus présente dans les études portant sur les langues, leurs acquisitions, leurs transmissions et leurs usages. L'emploi du concept de représentation en sociolinguistique est un emprunt aux sciences sociales, qui le tiennent elles-mêmes de la philosophie (Gueunier, 1997: 246). L'approche psychosociale a fortement influencé les recherches sociolinguistiques consacrées à ce sujet. Dans son article «Matériaux pour une approche des représentations sociolinguistiques », H. Boyer fait remarquer que : « les représentations de la langue ne sont qu'une catégorie de représentations sociales : même si la notion de représentation sociolinguistique, d'un point de vue épistémologique, fonctionne de manière autonome dans certains secteurs des sciences du langage » (Boyer, 1990 : 102). Pour cet auteur, le champ auquel renvoient les représentations est assez vaste, les représentations sociolinguistiques ne sont qu'une partie des représentations sociales. A ce titre, Denise Jodelet (1989: 43-36) considère les représentations sociales comme « une forme de savoir pratique reliant un sujet à un objet» ou encore comme "une forme de connaissance socialement élaborée et partagée, ayant une visée pratique et concourant à la construction d'une réalité commune à un ensemble social ».

5 L'analyse des représentations linguistiques est l'un des moyens privilégiés pour accéder aux différentes valeurs que l'on accorde aux langues. Ainsi, « comprendre comment les humains vivent sur le plan sociolinguistique, c'est comprendre comment ils se construisent et donc se représentent leurs univers sociolinguistiques » (Blanchet, 2003 : 301).

6 Notre recherche tâchera de cerner les représentations linguistiques en rapport avec le sentiment d'insécurité linguistique, car comme le note Calvet : « Il y a derrière chaque langue un ensemble de représentations explicites ou non, qui expliquent le rapport à cette langue sous forme d'attachement ou de répulsion » (Calvet, 1999 : 82). L'insécurité linguistique a longtemps intéressé des sociolinguistes francophones (M. Francard, N. Gueunier, P. Singy, D. Lafontaine, J-M Klinkenberg, W. Remysen, etc.). Ces chercheurs ont révélé des attitudes de dévalorisation de la langue française par les locuteurs issus des communautés francophones périphériques (Canada, Suisse, Belgique). Ces attitudes sont révélatrices d'un sentiment d'insécurité linguistique.

7 Dans sa conceptualisation, Michel Francard définit le concept comme «la prise de conscience, par les locuteurs, d'une distance entre leur idiolecte et une langue qu'ils reconnaissent comme légitime parce qu'elle est celle de la classe dominante» 
(Francard, 1993 : 13). Il souligne ailleurs (1997:171) que l'insécurité linguistique est la manifestation d'une quête de légitimité linguistique, vécue par un groupe social dominé, qui a une perception aiguisée aussi bien des formes linguistiques qui attestent sa minoration que des formes linguistiques à s'approprier pour assurer la promotion sociale. L'état de sécurité linguistique, par contre, «caractérise les locuteurs qui estiment que leurs pratiques linguistiques coïncident avec les pratiques légitimes, soit parce qu'ils sont effectivement les détenteurs de la légitimité, soit parce qu'ils n'ont pas conscience de la distance qui les sépare de cette légitimité » (Francard, 1997 :172).

\section{La langue berbère : de l'unité à la diversité}

La langue berbère (ou tamazight) occupe un vaste espace géographique constitué de plusieurs sphères linguistiques d'importance inégale, relativement isolées et séparées par des îlots arabophones plus ou moins importants. Cette langue est actuellement composée de plusieurs variétés présentes dans une dizaine de pays: Algérie, Maroc, Tunisie, Libye, Egypte, Niger, Mali, Burkina Faso, Mauritanie et îles Canaries.

9 En Algérie même, le berbère se présente sous forme de plusieurs variétés dont l'intercompréhension n'est pas toujours garantie. Said Chemakh (2011:19) distingue en Algérie environ neuf groupes linguistiques répartis sur des aires géographiques plus ou moins homogènes :

Aire kabyle (ou taqbaylit) au Nord (à l'est d'Alger),

Aire chaouie (ou tachawit) à l'est (sud-est Constantinois),

Aire chenouie (tachenwit) sur le Mont du Chenoua à l'ouest d'Alger,

Aire mozabite (Tamzabt) à Ghardaïa et dans les cités environnantes,

Aire touareg (Tamachaq) au sud, dans l'Ahaggar.

A côté de ces aires, se trouvent des îlots berbérophones moins importants tels que :

Tasenusit dans la sous-préfecture de Beni- Snous, département de Tlemcen,

Tazennatit dans les oasis du Gourara (Timimoun), département d'Adrar,

Tagergrent dans le département de Ouargla,

Tazennatit dans le Ksar de Bousemghoun, département de El-Bayadh.

La variation linguistique est tellement importante dans le domaine berbère que des linguistes s'interrogent encore aujourd'hui sur l'existence d'une ou de plusieurs langues berbères. Certains auteurs privilégient la thèse de l'unité profonde des parlers berbères, tout en reconnaissant l'existence de la diversité sur les plans phonétique, lexical et, quelquefois, morphosyntaxique. En revanche, d'autres chercheurs, en se basant sur le critère de l'intercompréhension qui est parfois difficile, adoptent une autre vision reconnaissant l'existence de plusieurs langues berbères.

En Algérie, la Kabylie est la communauté berbérophone qui contient le plus grand nombre de locuteurs (Chaker, 2004). En effet, le kabyle est parlé par deux tiers de la population berbérophone d'Algérie, lequel se compose de plusieurs variétés à référence régionale dont le tasahlit.

Les travaux en dialectologie dans le domaine berbère visant entre autres à mesurer la distance entre les variantes linguistiques, à regrouper les parlers...n'aboutissent pas toujours aux mêmes résultats. Par conséquent, plusieurs découpages des parlers de la région de Kabylie ont été proposés. Nous retenons, dans la présente recherche, trois grands espaces géographiques à savoir : la région du Sahel, la vallée de la Soummam et 
la Grande Kabylie. Il faut souligner que parmi les différentes variétés locales du kabyle, celle de la Grande Kabylie est la plus connue et bénéficie d'un certain prestige. Cette forme dominante du kabyle est "fondée à la fois sur le poids démographique, l'extension géographique, la représentation dans les études berbères et la production culturelle moderne - que ce soit la chanson ou l'écrit littéraire » (Chaker, $2004: 3$ ).

Revenons à la variété sur laquelle portera notre étude, le tasahlit. Il est à rappeler en effet que les études linguistiques qui étaient consacrées aux caractéristiques formelles de ce parler sont relativement rares. Que dire alors des études qui s'intéressent à la perception de ce régiolecte! Mener une recherche sur cette variété qui se trouve à l'extrême-est de la Kabylie est plus que nécessaire. A cet égard, Kamel Naït Zerrad affirme qu'un " gros travail de recherche est encore nécessaire pour la connaissance de tous les parler kabyles puisque certains ne sont encore que peu ou même pas du tout connus, en particulier les extrémités occidentale, et orientale du domaine " (Naït Zerrad, 2006 : 286).

Le Tasahlit est parlé dans la partie orientale de la Kabylie c'est-à-dire à l'est et au sudest de Béjaia, et s'étendant jusqu'aux frontières des wilayas de Jijel à l'est et de Sétif au sud-est (Berkaï, 2011 :95). En raison de son éloignement géographique, cette variété se distingue de ses pairs par différents traits linguistiques: phonétiques, morphosyntaxiques, lexicaux et parfois sémantiques. Sur ce point, Abdelaziz Berkaï souligne : « La dénomination même de tasahlit (de l'arabe sahel « littoral ») qu'on oppose généralement dans la région à taqbaylit (kabyle), renseigne sur la spécificité de ce parler dans l'ensemble du «kabyle » (Berkaï, 2011: 95). Sur le plan phonétique, le tasahlit comporte des traits phonétiques lui conférant une "identité phonétique" particulière. Ces spécificités ne se limitent pas uniquement au niveau phonique, elles sont également d'ordre morphosyntaxique. On peut citer ici le cas de la négation qui s'exprime en tasahlit de différentes façons: «ul-ula»; «ul-ani»; «ul-kra» ... au lieu de «ur-ara» («ne + verbe + pas ») dans l'ensemble du kabyle.

Les particularités linguistiques liées à ce parler posent quelques fois des problèmes d'intercompréhension lorsque les locuteurs de cette variété entrent en communication avec des locuteurs des autres régions de Kabylie. Kamel Naït Zerrad écrit à ce sujet : « Les sous-variétés contiguës sont linguistiquement très proches alors que celles situées aux extrêmes sont plus proches du dialecte avec lequel elles sont en contact (le chaoui pour la Kabylie extrême orientale) et l'intercompréhension entre elles est plus difficile » (Naït Zerrad, 2004 : 52).

Dans cet ordre d'idées, Abdelaziz Berkaï fait remarquer dans son article consacré au parler d'Aokas, que les différences de ce parler avec le kabyle occidental correspondent à des " rapprochements » avec d'autres parlers berbères comme le chleuh, le chaoui et même le touareg. En outre, ce même auteur souligne qu'il y a une opinion largement partagée dans la région selon laquelle la population d'Aokas, majoritairement maraboutique, serait d'origine chleuh.

\section{Notre enquête}

18 La récolte des données a été faite, dans un premier temps, à l'aide d'un questionnaire administré en ligne, composé de 34 questions ${ }^{1}$, certaines à choix multiples, d'autres ouvertes, réparties en deux catégories : la première portait sur le profil des enquêtés (âge, sexe, langue(s) maternelle(s), lieu de résidence, etc.); la deuxième, sur leurs 
attitudes à l'égard des différentes variétés linguistiques en présence dans la région de Kabylie. Nous nous sommes inspiré dans cette étude d'une approche utilisée par Pascal Singy (1997). Nous nous sommes d'ailleurs servi d'une grande partie de son questionnaire utilisé dans ses enquêtes sociolinguistiques sur le français parlé en Suisse romande et nous l'avons adapté à notre terrain de recherche car, même si les deux contextes peuvent présenter des similitudes, leurs différences restent toutefois indéniables.

Nous avons préféré administrer notre questionnaire en ligne afin d'obtenir le plus grand nombre de réponses et de toucher ainsi l'ensemble des régions où le tasahlit est pratiqué. De plus, les réponses obtenues à la suite du questionnaire auto-administré en ligne ont plus de chances d'être fiables et cela pour plusieurs raisons :

Les enquêtés n'ont pas à affronter le jugement de l'enquêteur. A notre avis, les enquêtés répondront plus facilement à un questionnaire à partir de leurs ordinateurs ou smartphones qu'en présence de l'enquêteur.

Les enquêtés peuvent prendre le temps qu'ils souhaitent pour y répondre. Ils n'ont pas le sentiment d'être pressés par l'enquêteur.

Par ailleurs, le recours à un questionnaire en ligne permet de contourner les risques d'erreurs lors de la saisie des réponses. Les données arrivent directement dans notre espace sur Google Forms. Pour diffuser notre questionnaire, nous avons créé un lien web que nous avons partagé sur les réseaux sociaux en ciblant notamment les pages portant les noms des villages de la région du Sahel.

21 Etant conscient des faiblisses que présente le questionnaire (possibilité de décalage entre la réalité et les pratiques déclarées...), notre enquête par questionnaire a été menée en parallèle avec d'autres approches qui permettraient d'en relativiser la portée par une confrontation de l'ensemble des résultats. Nous avons donc adopté une démarche fondée à la fois sur l'observation de situations de communication, sur l'analyse des questionnaires pour un aperçu général et sur une série d'entretiens semidirectifs effectués auprès de quelques locuteurs. Comme dans le questionnaire, l'entretien portait essentiellement sur le tasahlit et les rapports qu'entretiennent les locuteurs avec la variété en question.

\section{Population visée}

Les questionnaires ont été remplis par 162 participants issus des 9 communes de la région du Sahel : 27 informateurs sont d'Aokas, 19 de Darguina, 10 de Kherrata, 8 de Melbou, 20 de Souk El Tenine, 3 de Tamridjet, 31 de Taskriout, 14 d'Ait Smail et 8 de Tizi N'Berber. A cela s'ajoutent des enquêtés résidant dans d'autres régions de Béjaia ( 6 enquêtés habitent à Béjaia ville, 1 à Amizour, 1 à Tichy et 2 à Seddouk) et d'autres régions d'Algérie (3 informateurs résident à Alger, 2 à Sétif). L'administration du questionnaire en ligne nous a permis de recueillir des réponses d'enquêtés établis en Algérie mais aussi à l'étranger : en France (Paris), au Canada (Montréal) et aux USA (Boston et San Francisco).

23 L'enquête par questionnaire a permis d'interroger 130 hommes et 32 femmes. Notre échantillon présente, du point de vue du sexe, un net déséquilibre puisque notre questionnaire a été administré en ligne et de manière aléatoire. 

parle-t-on le mieux le kabyle à Béjaia?

\begin{tabular}{|l|l|l|}
\hline LA REGION & Nb. Cit. & Fréq. \\
\hline La ville de Béjaia & 13 & $8 \%$ \\
\hline La région du Sahel & 95 & $58,6 \%$ \\
\hline La vallée de la Soummam & 54 & $33,3 \%$ \\
\hline TOTAL OBS. & 162 & $100 \%$ \\
\hline
\end{tabular}

Soulignons d'emblée que les diverses variétés du kabyle ne sont pas perçues de la même façon en Kabylie. Certaines variétés sont valorisées voire survalorisées et d'autres, font face à une certaine dépréciation. La variété du kabyle parlée dans la région du Sahel est souvent dévalorisée et se trouve aujourd'hui dans une position inconfortable en raison notamment de ses particularités (visibles) lexicales, phonétiques et morphosyntaxique causant parfois des problèmes d'intercompréhension. C'est dans ce sens que nous avons 
posé une question fermée, invitant les enquêtés à citer un endroit à Béjaia (Kabylie), qui, selon eux, attestait le «meilleur kabyle ».

L'objectif de cette question était de recueillir l'avis des locuteurs concernant ces espaces de références sur le plan linguistique. L'examen des résultats pour l'ensemble de la population enquêtée fait apparaître que plus de la moitié des enquêtés est d'avis que c'est dans la région du Sahel que l'on parle mieux kabyle. Un autre point qui mériterait d'être signalé ici, et qui est en relation directe avec notre problématique (insécurité linguistique), est le fait que plus de $41 \%$ de répondants ont cité une région qui n'est pas la leur. C'est ce qui se dégage également de nos entretiens. Voyons cet extrait :

«Les autres kabyles pensent que le kabyle du Sahel est moins correct/ Ils font un mélange les gens du Sahel/ par exemple les gens de Kherrata/ la majorité parle la langue arabe parce qu'ils sont proches de Sétif et de Constantine/ Donc ils utilisent des mots arabes $»$.

Les enquêtés ayant produit un discours dévalorisant cette variété mettent souvent en avant la pureté de la langue. Le kabyle parlé dans la région du Sahel notamment dans les zones frontalières des wilayas arabophones (Jijel et Sétif) se caractérise par la présence de beaucoup termes empruntés de l'arabe. Ces emprunts sont perçus de manière négative, certains enquêtés sont allés jusqu'à qualifier ce parler de langue impure. La conception généralement répandue consiste donc à dire que la variété du Kabyle parlée dans cette région ne peut être la langue de référence comme le montre cet extrait :

«Les Kabyles du Sahel parlent un kabyle moins correct par rapport par exemple à celui qui est parlé à Tizi Ouzou/ par exemple chez nous/ les gens utilisent des termes de la langue arabe donc c'est pas du kabyle /Et des fois, on utilise le français / français cassé /mélangé avec du kabyle surtout les vieux / "

Comme dans le premier extrait, le tasahlit est considéré ici comme une variété qui résulterait d'un contact intense avec l'arabe. Il existe effectivement dans cette variété un nombre considérable de mots arabes qui donnent à ce parler une couleur particulière.

Il est utile de rappeler ici les travaux de Michel Francard sur la communauté française de Belgique (communauté dite "périphérique») qui se définit en permanence par rapport au centre qu'est Paris. Cet auteur parle de "la sujétion linguistique à la France » c'est-à-dire que le français de Paris est généralement considéré comme la variété légitime par rapport à laquelle les autres variétés sont dans une situation de subordination. Il y a là, nous croyons, des parallèles à établir avec la région du Sahel en Kabylie car bon nombre d'enquêtés ont tendance à hiérarchiser les diverses variétés du Kabyle et situent le "bon kabyle » en dehors de leur région. Nous avons donc affaire à l'insécurité linguistique portant sur la forme linguistique. En témoignent ces propos recueillis auprès de l'un des locuteurs interrogés :

"Je pense que nous parlons moins bien que les autres kabyles/même si je suis de là-bas /je préfère tasahlit /mais la vérité / tasahlit est moins correcte / ils utilisent des mots arabes / peff français / il y a un mélange important / Le kabyle de Tizi Ouzou est plus raffiné / mais pas celui de Béjaia / et encore moins celui du Sahel ».

Dans les travaux de beaucoup de sociolinguistes francophones, l'insécurité linguistique est présentée comme une "quête de légitimité linguistique ». C'est le sentiment que ressent un individu lorsqu'il pense ne pas pratiquer une langue ou variété de langue légitime ou prestigieuse. La réalité décrite par ces linguistes ne diffère pas tellement de 
ce que nous avons pu observer dans notre terrain d'enquête où certains locuteurs entretiennent des sentiments négatifs à l'encontre de leur propre régiolecte. Il s'agit là de l'une des principales facettes de l'insécurité linguistique. Cette dernière prend racine surtout dans un contexte de pluralité c'est-à-dire dans un environnement où plusieurs langues et/ou variétés de langue sont pratiquées.

A votre avis, le parler kabyle le plus raffiné (beau, élégant, joli ...) est celui de :

\begin{tabular}{|l|l|l|}
\hline LA REGION & Nb. Cit. & Fréq. \\
\hline La région de la grande Kabylie & 38 & $23,5 \%$ \\
\hline Région du Sahel & 88 & $54,3 \%$ \\
\hline La région de la vallée de la Soummam & 36 & $22,2 \%$ \\
\hline TOTAL OBS. & 162 & $100 \%$ \\
\hline
\end{tabular}

Toujours dans le but de révéler d'autres facettes de l'insécurité linguistique, nous avons demandé à nos informateurs, par le biais d'une question fermée, de citer le parler kabyle le plus raffiné (beau, élégant, joli...). Un accent raffiné n'était pas autre chose qu'un accent qu'on reconnaissait empreint d'élégance, de beauté ou encore de distinction (Singy, 1997).

Il ressort de notre enquête que $54 \%$ des enquêtés considèrent le tasahlit comme le parler le plus raffiné. On peut aussi signaler qu'il y a plus de $45 \%$ de répondants qui ne tiennent pas le tasahlit comme étant la variété du kabyle la plus raffinée. Ces résultats montrent clairement qu'une partie de la population du Sahel est convaincue qu'il y a des variétés du kabyle plus raffinées que d'autres (Singy, 1997). En témoignent ces propos recueillis lors des entretiens :

"A mon avis / il y a des endroits où on parle mieux kabyle / parce dans le parler du Sahel il y a des mots vraiment compliqués / parfois il y a même des mots bizarres qui n'ont pas de sens /c'est mon avis ».

«Moi je pense que/ c'est la variété de Tizi Ouzou qui est la plus valorisée/ les gens de Tizi utilisent des termes qui ont une relation avec tamazight / berbère /les vrais Kabyles/Nous / on utilise des mots arabes/ par exemple/ nous /pour désigner la porte on dit «Lbab » / c'est un mot qui vient de l'arabe ».

On considère souvent les locuteurs de la Kabylie du Durdjura (appelée également Grande Kabylie) comme les héritiers du kabyle originel "c'est le vrai kabyle chez eux». Cette variété est considérée par certains enquêtés comme supérieure à la variété parlée dans leur région. Cette attitude s'explique par le fait que cette variété est la plus présente à la télé, à la radio...mais également dans la création artistique. D'autres locuteurs ont exprimé leurs préférences pour la variété du kabyle parlée à Bejaïa ville. On a considéré cette variété comme étant "plus belle ", "plus douce ", "plus raffinée ", etc. Par comparaison, le tasahlit est considéré comme "bizarre", "moche», etc. Observons les propos ci-dessous récoltés auprès de l'une de nos informatrices où l'on peut observer une dépréciation du régiolecte, l'enquêtée en question affirme sans ambigüité qu'il ne s'agit pas d'un kabyle raffiné :

"J'ai toujours pensé que la variété du kabyle parlée à Béjaia est plus raffinée que tasahlit /Il m'arrive souvent d'assister à des fêtes dans la région du Sahel /et je ne 
parle pas tasahlit /même si je suis de là-bas/ Mes cousins et cousines me disent toujours / " pourquoi tu ne parles pas ta langue (rire) »/» tu l'as changée »/ «c'est pas bien »/On me fait beaucoup de reproches par rapport à ma façon de parler/ Mais moi /je leur dis/ ben / je leur dis que je parle comme je veux / que je considère la langue bougiote plus belle /plus raffinée plus .../ »

\begin{tabular}{|l|l|l|}
\hline AVIS & Nb. Cit. & Fréq. \\
\hline OUI & 118 & $72,8 \%$ \\
\hline NON & 44 & $27,2 \%$ \\
\hline TOTAL OBS. & 162 & $100 \%$ \\
\hline
\end{tabular}

42 A la différence des précédentes questions, notre objectif ici n'est pas de mettre en évidence la tentation de la population enquêtée à dévaloriser son parler. Notre question invitait cette fois-ci les enquêtés à nous dire s'ils repèrent dans la pratique linguistique de leurs semblables des éléments susceptibles de traduire une tendance à la dévalorisation de ladite variété.

Ce temoignage nous renseigne sur une attitude paradoxale : les locuteurs attribuent la meilleure qualité de langue aux variétés parlées en dehors de leur région mais, en même temps, ils associent des valeurs négatives à ces mêmes variétés lorsqu'elles sont employées par un des leurs, qu'ils accusent de vantards et de frimeurs voire de « renégats ». Ainsi, « d'une part la culture ambiante indique un modèle linguistique aux individus, d'autre part, elle leur enjoint de ne pas l'adopter» (Moreau, 1999). Cette insécurité linguistique est répandue à travers cette région en partie à cause de l'opinion populaire négative qui y circule. La majorité des enquêtés rapportent s'être déjà fait dire qu'ils parlent mal ou bien qu'ils ont un accent qui est " mauvais ». Ces locuteurs ont, en quelque sorte, intériorisé l'idée que leur kabyle n'est pas raffiné. D’ailleurs, quelques enquêtés se sont montrés hostiles à la perspective d'une intégration des mots de tasahlit dans les dictionnaires berbères et même dans l'enseignement de la langue berbère.

est l'existence d'un malaise par rapport à la variété de langue pratiquée. On voit chez certains locuteurs la conscience d'une distance entre la langue qu'ils parlent et la langue qu'ils perçoivent comme légitime. Ils comparent leur propre variété du kabyle à celles parlées dans les autres régions qu'ils estiment plus légitimes parce qu'elles sont " comprises par tout le monde ». Une partie de la population enquêtée vit alors un certain complexe linguistique (majorité vs majorité). Il y a donc des centres et des périphéries, mais de toute évidence, pour beaucoup de locuteurs du Sahel, leur région d'origine représente toujours la périphérie.

Est-il vrai que certains locuteurs du Sahel essaient d'effacer leur accent lorsqu'ils sont devant les autres kabyles?

On s'est donc attaché à demander aux enquêtés s'il existe ou non, d'après leurs expériences personnelles, des locuteurs de leur région qui essayent d'effacer leur accent. Les réponses à cette question se présentent, pour la population enquêtée, de la manière suivante: une très large majorité des personnes interrogées (presque $73 \%$ ) 
s'accordent à penser qu'il existe des locuteurs de leur région qui s'appliquent à perdre leur accent, $27 \%$ seulement expriment le contraire. La même réalité se dégage des entretiens comme on peut le lire dans cet extrait :

«Je crois que le sentiment d'infériorité linguistique est présent chez beaucoup d'individus/ par exemple lorsqu'ils parlent/ ils utilisent une autre variété/ J'ai remarqué que lorsqu'ils viennent par exemple à Béjaia / ils parlent Tabejawit / Ils ont en quelque sorte un complexe vis-à-vis de leur langue / Ils considèrent les autres variétés comme plus prestigieuses/ supérieures...»

Il ne fait nul doute que le sentiment d'insécurité est ressenti davantage lorsque les locuteurs se retrouvent dans des espaces sociaux où leur variété est minoritaire. Par ailleurs, ces mêmes locuteurs peuvent se sentir en sécurité linguistique lorsqu'ils sont dans leur région avec leurs semblables. Certains contextes imposent aux locuteurs de cette variété de se conformer aux pratiques linguistiques dominantes. C'est ce qui ressort dans le témoignage suivant:

«Je parle avec mes voisines en bougiote/ Presque c'est la majorité qui fait ça/ par exemple on a d'autres voisins/ ils sont de Kherrata /à l'extérieur ils parlent le bougiote normal / Les gens du Sahel effacent leur accent plus que les autres / surtout ceux qui viennent s'installer à Béjaia /parce que eux même n'aiment pas leur langue/ d'après mon expérience / je connais beaucoup de gens qui sont du Sahel et qui n'aiment pas leur langue / ils effacent et changent de langue / ils sont pas fiers /"

Les recherches sociolinguistiques ont révélé que les femmes constituent un groupe particulièrement sensible aux modèles linguistiques de prestige. Pierre Bourdieu (1982) constate, à cet égard, que les hommes sont moins attirés par les façons de parler légitimes que les femmes (surtout les plus jeunes et les plus scolarisées). C'est ce qui se dégage de notre enquête notamment lors des entretiens. Nos enquêtés ont tous en tête des exemples de personnes (de femmes en particulier) qui ont nourri un sentiment d'infériorité linguistique et qui ont décidé à un moment donné de leur vie de perdre leur accent au profit d'une autre façon de parler considérée comme plus prestigieuse. Observons ce témoignage :

« ma cousine a effacé complètement sa langue /elle a étudié / je pense trois ans ici à l'université /elle a changé de langue/lorsqu'on parle avec elle tasahlit/elle se moque de nous /surtout de moi /elle me dit /» 5 ans à l'université et tu n'arrives pas à changer / elle parle donc le bougiote /et même le parler de Tizi Ouzou / puisqu'elle fréquente beaucoup les filles de Tizi /mais notre langue elle l'a effacée complètement/quand elle rentre chez elle les weekend /elle parle également le bougiote/»

Les raisons qui conduisent à l'abandon de l'emploi de cette variété sont nombreuses et varient selon les contextes et les individus. Nous pouvons tout de même dégager un trait commun: en général, ce sont des gens qui, pour la plupart, ont une forte conscience linguistique, c'est-à-dire qu'ils sont conscients de l'écart qui sépare leur variété des autres variétés du kabyle. Les locuteurs de tasahlit prennent réellement conscience de leur différence une fois qu'ils sont en contact avec les autres locuteurs kabylophones. Pour les jeunes universitaires, c'est surtout à l'université qu'ils découvrent réellement à quel point leur variété est différente. Il est frappant de constater que certains étudiants se sont même sentis rejetés durant les premières années de leur cursus universitaire :

«Moi je suis plus à l'aise avec les gens du Sahel que les autres / parce les autres se font des préjugés/ en première année / j'étais la seule du groupe qui vient du Sahel 
/ J'ai carrément voulu changer de groupe / la langue /elle pose problème des fois /

on m'a un petit peu rejeté / » marginalisation par quelques locuteurs et disent recevoir des commentaires négatifs de la part de gens par rapport à leur façon de parler. Si les locuteurs de ce régiolecte sont par conséquent tentés par cet effacement d'accent, c'est principalement parce qu'ils subissent beaucoup de pression. La majorité de nos enquêtés $(80 \%)$ disent avoir déjà reçu des remarques par rapport à leur façon de parler. Ils ont déjà subi des expériences où ils se sont sentis comme si leur kabyle n'était "pas assez bon", ou que leur langue était "trop rapide", "bizarre", "compliquée», etc. À en juger par ce commentaire épilinguistique :

« Les premiers temps/j'ai rencontré beaucoup de difficultés /on se moquait de moi en disant que je suis Tasahlit/ que j'ai un accent bizarre/vous imaginez /dès le primaire /les enfants ont déjà ce jugement négatif envers les gens du Sahel/les élèves me disent/ "tu es du Sahel»/» tu ne parles pas bien»/» ta langue est bizarre ».../ »

L'exemple ci-dessus montre, à lui seul, le poids des jugements portés sur cette variété. Nous comprenons alors qu'il existe un lien de causalité entre les particularités formelles (phonétiques, lexicales et morphosyntaxiques) de cette variété et sa perception par ses propres locuteurs et aussi par les autres locuteurs. En d'autres termes, les locuteurs sont plus enclins à éprouver de l'insécurité linguistique lorsque les particularités linguistiques de la variété qu'ils parlent sont importantes. Certains traits spécifiques, notamment phonétiques, deviennent dévalorisants.

Le même phénomène peut être observé chez quelques femmes issues de cette région de Kabylie, mariées à des hommes parlant une autre variété du kabyle. Ces femmes-là ont dû abandonner leur accent afin de se faire accepter et de s'intégrer ainsi au sein de la belle famille. Voilà donc des signes tangibles de cette insécurité :

«J'ai une cousine qui s'est mariée ici à Béjaia/ elle est du Sahel /mais elle est mariée à un bougiote/ ses enfants ne parlent jamais tasahlit/ même elle/ elle ne parle jamais tasahlit / même lorsqu'elle part chez ses parents /elle parle le bougiote / et parfois ses belles sœurs / elles disent oh la femme de... /elle est du Sahel/ elle parle tasahlit/genre c'est toujours mal vu »

La perception de tasahlit par ses propres locuteurs dépend ainsi des jugements portés par les autres locuteurs comme l'affirme Cécile Canut (1998) : « l'activité épilinguistique se nourrit [aussi] des discours ambiants ». Autrement dit, les locuteurs de la variété de tasahlit sont très conscients non seulement des spécificités de leur parler et des hiérarchies qui sont construites, mais également du regard des autres, du discours qui circule sur leur variété et sur les préjugés dont elle fait l'objet. C'est pourquoi nous avons interrogé nos informateurs sur l'image qu'ont les autres kabyles en général de cette variété (Leblanc, 2010). Il semble y avoir consensus parmi ces locuteurs, la majorité d'entre eux (soit $73 \%$ ) soulignent que la variété du Sahel est souvent perçue de façon négative en Kabylie.

51 En d'autres termes, les prises de position adoptées par les locuteurs kabylophones des autres régions par rapport à la variété de tasahlit ne peuvent qu'avoir un effet sur les représentations que se font les locuteurs de la variété en question, à la longue, générer une certaine insécurité (Leblanc, 2010). Cette dernière exerce inévitablement un impact sur les pratiques concrètes. L'effacement de l'accent est, sans doute, l'un des indices les plus saisissants du sentiment d'insécurité linguistique. Dans l'extrait ci-dessous, l'un de 
nos enquêtés nous raconte à quel point ce phénomène d'effacement de l'accent est important et touche même les petits enfants :

»Peut-être que cette variété va disparaitre chez les générations à venir /même les enfants / y en a ceux qui n'aiment pas tasahlit/ par exemple/ j'ai une nièce / elle habite dans la région du Sahel/ elle a 8 ans/elle vient chez nous durant les vacances /donc elle a des amies /des voisines qui ont des petites fillettes de son âge/ donc elle sort avec elles pour jouer et tout ça/elle essaye toujours de parler comme elles /elle me dit /je n'aime pas ma langue / et après /elle me dit tasahlit on comprend pas ce que tu veux dire /joue comme nous/ elle a déjà ce sentiment/elle commence déjà à dévaloriser sa langue "

Cet exemple sur l'insécurité est frappant, il montre l'ampleur de ce sentiment de malaise des locuteurs par rapport à leurs usages. Le sentiment d'insécurité linguistique a des conséquences sur les pratiques linguistiques effectives des locuteurs. Dans un entretien qu'elle a accordé en 2015 au Musée Canadien de Langues, Annette Boudreau affirme que "Les discours négatifs sur les pratiques linguistiques des locuteurs sont reliés à une forme de rejet de l'autre et que les conséquences de ces discours sont énormes comme : certaines personne n'osent plus parler ». Pour nos informateurs, ces intimidations linguistiques ${ }^{2}$ sont présentes dans divers contextes (à l'école, à l'hôpital, dans la rue, au marché, à l'université, etc.) les poussant ainsi à adopter une autre façon de parler. Arrêtons-nous à ce témoignage :

«la famille de son mari sa belle-famille/ ils n'acceptent pas sa langue/ depuis qu'elle est fiancée elle parle le bougiote/même ses enfants/ ils parlent jamais tasahlit/ même avec sa famille à elle/ elle ne parle pas en tasahlit/ Tout le monde la déteste à cause de ça / ils disent /pourquoi / genre elle est arrogante/ elle a changé sa langue / par contre/ y en d'autres c'est le même cas/ elles sont mariés avec des bougiote /mais elles parlent tasahlit avec leurs enfants /ça dépend des cas /elles parlent les deux langues le bougiote et tasahlit /chez elles elles parlent tasahlit/ chez les autres elles parlent le bougiote ».

Il existe bien entendu plusieurs cas de figures, certains locuteurs effacent leur accent dans toutes les situations de communication alors que d'autres adaptent leurs façons de parler en fonction de leurs interlocuteurs. Dans cette perspective, le sociologue français Pierre Bourdieu avance les concepts de langue légitime et langue vernaculaire. La première est dotée de valeurs symboliques, la deuxième, en revanche, est empreinte de valeurs de solidarité. Ainsi, beaucoup de locuteurs n'admettent pas le fait que des personnes de leur région s'expriment dans une autre variété surtout en présence de leurs pairs. L'usage d'une variété autre que le tasahlit sera en effet interprété comme une "vantardise".

Nous pouvons dire que beaucoup de locuteurs de cette variété ont fini par céder à la pression résultant du discours dominant dévalorisant leur variété. Le meilleur exemple de cette dévalorisation est l'absence de cette variété dans les médias. Les journalistes, y compris ceux qui sont issus de cette région, choisissent de s'exprimer dans une autre variété que la leur. À cela s'ajoute le milieu artistique au sein duquel se produit le même phénomène. Toutefois, on observe ces derniers temps, ici et là, des chanteurs (si peu soient-ils) qui ont fait le choix de chanter exclusivement dans cette variété. Il s'agit en quelque sorte d'un contre-discours sur la langue qui se manifeste par le recours à la variété stigmatisée dans le but de renverser les stigmates (Leblanc, 2010).

Comme on vient de le voir, les manifestations d'insécurité linguistique chez la population enquêtée sont multiples et diverses. La qualité de langue est attribuée par certains de nos informateurs à des variétés qui sont parlées en dehors de leur région. 
Ils considèrent la variété linguistique qu'ils parlent comme étant moins correcte et donc non-légitime en comparaison à celles qui sont parlées en dehors de leur région. Il est clair qu'une telle attitude exerce inéluctablement un impact sur les pratiques linguistiques, c'est ce qui ressort d'ailleurs de notre enquête. Nos informateurs nous ont livré nombre de témoignages concernant des personnes de leur entourage qui ont abandonné leur variété de langue au profit des variétés du kabyle qu'ils jugent plus prestigieuses et/ou moins marquées.

Au fil de cette étude, nous avons tenté d'expliquer les raisons qui sont à l'origine de l'apparition de l'insécurité linguistique dans les milieux minoritaires à l'instar du nôtre. En effet, nous avons pu constater que les discours dominants (souvent négatifs) sur la variété parlée dans la région étudiée influencent la façon dont les locuteurs perçoivent leur variété et leurs pratiques linguistiques en général. La conscience de l'existence de diverses façons de parler -ou la conscience de parler une variété de langue différenteconstitue un des facteurs pouvant participer à l'apparition du sentiment d'insécurité linguistique. Autrement dit, les traits linguistiques spécifiques à cette variété deviennent des éléments déclencheurs de l'insécurité.

Enfin, il est à souligner qu'il existe de réelles disparités dans les rapports que les locuteurs interrogés entretiennent avec leur régiolecte. De fait, c'est exclusivement pour des considérations d'ordre pratique que l'accent n'a été mis ici que sur la catégorie de locuteurs ayant produit un discours dépréciatif à l'encontre de tasahlit. Beaucoup de nos informateurs ont produit des discours dont le contenu invite à penser qu'ils cultivent des représentations positives de leur variété. L'attachement au régiolecte, compris en termes identitaires, peut offrir à ces locuteurs une certaine sécurité linguistique.

1 -Vous êtes: Une femme Un homme

2 - Votre âge: Moins de 30 ans entre 30 et 50 ans plus de 50 ans

3-Dans quelle commune résidez-vous actuellement?

4 - Dans quelle région avez-vous passé la plus grande partie de votre vie?

Région du Sahel La vallée de la Soummam

La Grande Kabylie Autre (précisez)

5 - Quelle est votre langue maternelle?

kabyle arabe Autre (précisez)

6- Si vous répondez par « kabyle », il s'agit de quelle variété ?

variété du Sahel variété de la vallée de la Soummam

variété de la Grande Kabylie (Tizi-Ouzou)

7 - Selon vous, le kabyle parlé dans la région du Sahel est plutôt de qualité :

bonne médiocre très bonne moyenne

$8-$ On dit qu'il y a des endroits où l'on parle mieux le kabyle qu'ailleurs. Selon vous, où parle-t-on le mieux le kabyle à Béjaia?

Région du Sahel La vallée de la Soummam La ville de Béjaia 
9 - A votre avis, le jugement que portent les Kabyles en général sur le parler du Sahel est plutôt : négatif positif ils ne portent pas de jugement

10 - Le jugement que portent les locuteurs du Sahel sur leur parler est plutôt :

négatif positif ils ne portent pas de jugement

11 - A votre avis, le parler kabyle le plus raffiné (beau, élégant, joli ...) est celui de :

La région de Sahel La région de la vallée de la Soummam La région de la Grande Kabylie

$12-$ Est-ce que vous aimez votre accent?

oui beaucoup plutôt oui plutôt non non pas du tout

13-Avez-vous l'impression que les Kabyles du Sahel pensent parler un kabyle moins correct que les autres kabyles? oui non

$14-Y$ a-t-il des locuteurs du Sahel qui essaient de perdre leur accent ?

oui non

15 - Quand vous entendez des gens parler avec un accent du Sahel, êtes-vous :

fier indifférent gêné amusé agacé

16 - Est-il vrai que certains locuteurs du Sahel essaient d'effacer leur accent lorsqu'ils sont devant les autres kabyles? oui non

17 - Avez-vous l'impression que les Kabyles du Sahel pensent parler un kabyle moins correct que les autres parlers kabyles? oui non

18- On peut très bien parler le kabyle avec un accent du Sahel :

Pas du tout d'accord Plutôt pas d'accord

Plutôt d'accord Tout à fait d'accord

19-A votre avis, les gens du Sahel parlent plutôt :

mieux que les autres kabyles moins bien que les autres kabyles

aussi bien que les autres kabyles

20- Est-il plus correct de dire :

ur truhuy ara ul truhuy ula les deux sont également corrects

21- L'école doit enseigner aux enfants des mots de la variété « tasaḥlit »

Pas du tout d'accord Plutôt pas d'accord

Plutôt d'accord Tout à fait d'accord

22-Les mots de la variété « tasahlit » devraient figurer dans les dictionnaires berbères : Pas du tout d'accord Plutôt pas d'accord

Plutôt d'accord Tout à fait d'accord

23- Utilisez-vous quelquefois des mots de la variété « tasaḥlit » pour affirmer votre identité et votre appartenance à cette région? oui non 
24-Quels sont les éléments qui permettent, selon vous, de reconnaitre plus ou moins aisément la façon de parler qu'ont les locuteurs du Sahel ?

Lexique débit accent syntaxe

25- Surveillez-vous votre façon de parler quand vous parlez à des locuteurs issus des autres régions de Kabylie? oui non

-Pourriez-vous dire pourquoi vous surveillez votre façon de parler?

26- Avez-vous eu déjà des remarques sur votre façon de parler?

oui non, -si "oui", merci de préciser le type de remarques qu'on vous a faites.

27- Avez-vous déjà effacé votre accent en dehors de votre lieu de résidence ?

oui non, -si oui, pourquoi?

28-Quand vous parlez kabyle et arrive une autre personne qui parle une autre variété, continuerez-vous de parler dans votre variété?

oui non - Pourriez-vous nous dire pourquoi?

29- Lorsque vous êtes en présence d'un kabyle s'exprimant dans une autre variété que la vôtre, quelle variété du kabyle choisirez-vous?

variété de la Soummam variété de la Grande Kabylie

variété du Sahel autre (précisez)

30-Quelle est la variété du kabyle que vous préférez le plus

variété de la Soummam variété de la Grande Kabylie

variété du Sahel Aucune variété

31-Comment évaluez-vous votre façon de parler kabyle?

bonne moyenne mauvaise

32- Avez-vous été un jour jugé (e) négativement en raison de votre accent pour l'obtention d'un poste de travail, d'un logement, etc. ?

oui non, si oui, pourriez-vous raconter cette expérience?

33- Avez-vous été un jour stigmatisé (e) ou rejeté (e) en raison de votre manière de parler kabyle? oui non, si oui, pourriez-vous raconter cette expérience?

34- Est-ce un jour quelqu'un s'est moqué de vous en raison de votre façon de parler kabyle? oui non

BIBLIOGRAPHIE

BERKAï, Abdelaziz., « Les spécificités morphosyntaxiques du parler kabyle d'Aokas », Asinag, $\mathrm{n}^{\circ}$ 6, Rabat, 2011, p. 95-114. 
BLANCHET, Philippe., « Contacts, continuum, hétérogénéité, polynomie, organisation « chaotique », pratiques sociales, interventions ... quels modèles ? Pour une (socio)linguistique de la « complexité », Cahiers de sociolinguistique, $\mathrm{n}^{\circ}$ 8, Rennes, 2003, p. 279-308.

BLANCHET, Philippe., Discriminations : combattre la glottophobie, Paris, Éd. Textuel, coll. Petite Encyclopédie critique, 2016.

BOUDREAU, Annette., " La construction des représentations linguistiques : le cas de l'Acadie ", Revue canadienne de linguistique, $n^{\circ}$ 54, 2009, p. 439-459.

BOURDIEU, Pierre., Ce que parler veut dire, l'économie des échanges linguistiques, Paris, Fayard, 1982.

BOYER, Henri., « Matériaux pour une approche des représentations sociolinguistiques. Eléments des définitions et parcours documentaires en diglossie ", Langue française, $\mathrm{n}^{\circ} 85,1990$,

p. 102-124.

CALVET, Louis-Jean., pour une écologie des langues du monde, Paris, Plon, 1999

CHAKER, Salem., « Kabylie : La langue », Encyclopédie berbère, 26 | Judaïsme - Kabylie, Edisud, Aix-enProvence, 2004, p. 4055-4066.

CHEMAKH, Saïd., «L'Aménagement de tamazight (milieu algérien) : Etat des lieux, critiques et propositions ", timsal n Tamazight, $n^{\circ}$ 1, Septembre 2011, p. 15-28.

FRANCARD, Michel., L'insécurité linguistique en Communauté française de Belgique, Bruxelles, Collection "Français et Société", n 6, Service de la langue française, 1993.

FRANCARD, Michel., L'insécurité linguistique dans les communautés francophones périphérique, Louvain, Cahiers de l'Institut de Linguistique de Louvain, 1993.

FRANCARD, Michel., «Insécurité linguistique », dans MOREAU MARIE-LOUISE (dir.), Sociolinguistique : concepts de base, Liège, Mardaga, 1997, p. 170-176.

GUEUNIER, Nicole., « représentations linguistiques », dans MOREAU MARIE-LOUISE (dir.), Sociolinguistique : concepts de base, Liège, Mardaga, 1997, p. 246-251.

HAFEZ, Stéphane-Ahmad., "L'insécurité linguistique au Liban : le cas des futurs enseignants de français ». Actes du colloque de Dijon mai 2011, publiés en 2012.

JODELET, Denise., Les représentations sociales, Paris, PUF, 1989.

NAÏT-ZERRAD, Kamel., « Kabylie : Dialectologie », Encyclopédie berbère, 26 | Judaïsme - Kabylie, Aix-enProvence, Edisud, 2004, p. 4067-4070.

NAÏT-ZERRAD, Kamel., « Variation, standardisation et enseignement », in Actes du colloque international sur l'enseignement des langues maternelles, les 23, 24, et 25 mai 2003, UMMTO publiés en 2006.

LEBLANC, Matthieu., » Le français, langue minoritaire, en milieu de travail : des représentations linguistiques à l'insécurité linguistique », Nouvelles perspectives en sciences sociales, Volume $6, \mathrm{~N}^{\circ} 1$, Décembre, 2010, p. 17-63.

MOREAU, Marie-Louise., « La pluralité des normes dans la francophonie », 1999.

SINGY, Pascal., L'image du français en Suisse romande : une enquête sociolinguistique en Pays de Vaud, Paris, L'Harmattan, 1997.

vorsin, Camille., «Attitudes des francophones du Nouveau Brunswick à l'égard du chiac ", Travaux neuchâtelois de linguistique, $\mathrm{n}^{\circ}$ 64, 2016, p. 101-119. 


\section{ANNEXES}

Questionnaire

\section{NOTES}

1. Pour des raisons pratiques, nous nous sommes limité à l'analyse des questions les plus pertinentes en lien étroit avec la problématique traitée.

2. Philippe Blanchet développe dans son livre (2016) qu'il a consacré aux discriminations linguistiques le concept de glottophobie qu'il définit ainsi « le mépris, la haine, l'agression, le rejet, l'exclusion, de personnes, discrimination négative effectivement ou prétendument fondés sur le fait de considérer incorrectes, inférieures, mauvaises certaines formes linguistiques »

\section{RÉSUMÉS}

Notre contribution fait état des résultats d'une enquête sociolinguistique sur la variété du kabyle parlée dans les régions de la Kabylie maritime, ou ce que l'on appelle communément « Le Sahel ». Elle se donne pour objectif de réfléchir au phénomène d'insécurité linguistique et d'examiner, grâce à l'étude des représentations linguistiques, ses diverses manifestations chez des locuteurs de tasahlit.

The study reports the results of a sociolinguistic survey on the variety of Kabylian spoken in the regions of Kabylia Maritime or what is commonly called "The Sahel". Then we aim to analyze the phenomenon of linguistic insecurity through the study of linguistic representations, its various manifestations among tasahlit speakers.

INDEX

Mots-clés : insécurité linguistique, représentation des langues, légitimité linguistique, minorité linguistique, tasahlit

Keywords : linguistic insecurity, language representation, linguistic legitimacy, linguistic minority, tasahlit

\section{AUTEUR}

\section{BACHIR BESSAI}

Laboratoire LESMS Faculté des lettres et des langues Université de Bejaia, 06000 Bejaia, Algérie 